$32^{\text {nd }}$ Winter School of Theoretical Physics,

Karpatz, Poland, February 19-29, 1996

Solid State Physics: From Quantum Mechanics to Technology

(to be published in Lecture Notes in Physics, Springer 1996)

\title{
CHARGE DYNAMICS IN CUPRATE SUPERCONDUCTORS
}

\author{
E. Tutiš \\ Institute of Physics of the University of Zagreb, \\ P.O.B. 304, Zagreb, Croatia \\ H. Nikšić and S. Barišić \\ Department of Physics, Faculty of Sciences, \\ P.O.B. 162, Zagreb, Croatia
}

\begin{abstract}
In this lecture we present some interesting issues that arise when the dynamics of the charge carriers in the $\mathrm{CuO}_{2}$ planes of the high temperature superconductors is considered. Based on the qualitative picture of doping, set by experiments and some previous calculations, we consider the strength of various inter and intra-cell charge transfer susceptibilities, the question of Coulomb screening and charge collective modes. The starting point is the usual $p$ - $d$ model extended by the long range Coulomb (LRC) interaction. Within this model it is possible to examine the case in which the LRC forces frustrate the electronic phase separation, the instability which is present in the model without an LRC interaction. While the static dielectric function in such systems is negative down to arbitrarily small wavevectors, the system is not unstable. We consider the dominant electronic charge susceptibilities and possible consequences for the lattice properties.
\end{abstract}

\section{Introduction}

A decade of experimental and theoretical research indeed showed that the physics of the high temperature copper-oxide superconductors is both complex and intriguing. One major source of complexity lies in the fact that the electronic correlations in these materials are strong. The antiferromagnetism in undoped $\mathrm{LaCuO}_{4}$ and $\mathrm{YBa}_{2} \mathrm{Cu}_{3} \mathrm{O}_{6}$ is clear evidence of that. A second source may be found in experiments that suggest that the electron-phonon interaction may be strong as well. As far as the effects of strong electron-electron interactions on the electronic properties of doped 
superconducting materials are concerned, two different viewpoints exist in general. From the first point of view, the spin correlations, although much weakened on doping, still dominantly determine the carrier dynamics. From the second viewpoint the superconducting cuprates are doped charge transfer insulators, in which the tendency towards spin ordering may be an important, but still secondary, effect. This second approach focuses on the charge degrees of freedom and charge fluctuations, and the effects that they may have on superconductivity [1, 2], electronic properties in the metallic phase, and the crystal lattice dynamics [3]. Some of these issues are the subject of this lecture. We consider dominant static charge susceptibilities, the collective modes and the dielectric properties for the three dimensional system of copper-oxide planes. The features that emerge are related to the properties of these materials observed in experiments. In particular, these are the anomalies found in the modes of oscillations of in-plane oxygen atoms, the apex oxygen position and movement and the incommensurate and commensurate deformations in the direction parallel to planes. In this lecture we consider the system that is close to the doped charge transfer insulator. However, some of the conclusions remain qualitatively valid away from this regime. This is in particular true for the foregoing discussion related to the oxygen-oxygen charge transfer modes and the discussion regarding the frustrated phase separated instability.

\section{The p-d model with long range Coulomb interaction}

The presence of $\mathrm{CuO}_{2}$ planes is the mayor feature of all cuprate high temperature superconductors. The electronic hopping between the planes is relatively small. The 'insulating' layer between the planes may be composed by atoms of various elements. Another generic feature, as shown by many experiments (e.g., EELS, X ray absorption, photoemission), is that on doping the excess holes mostly populate the $p_{x, y}-\sigma$ orbitals of the in-plane oxygen atoms. These orbitals further hybridize with some, mainly $d_{x^{2}-y^{2}}$, orbitals on the copper atoms. Together with the fact that correlation effects are important for the in-plane dynamics of electrons, this constitutes the basis of the $p$ - $d$ model.

The original $p$ - $d$ model [5, [1] consists of the tight-binding part and the part accounting for the short range Coulomb interaction,

$$
H_{p d}=H_{0 t b a}+H_{S R C}
$$

In the tight-binding part the orbital energy levels $\left(\varepsilon_{d}, \varepsilon_{p}, \Delta_{p d} \equiv \varepsilon_{p}-\varepsilon_{d}\right)$ are specified and various hybridization terms (copper-oxygen, $t_{0}$, oxygen-oxygen, $t^{\prime}$, etc.) included. $H_{S R C}$ contains the terms describing the Coulomb repulsion on the copper, $U_{d}$, and oxygen site, $U_{p}$, as well as the terms describing the interaction between electrons on neighbouring sites (copper-oxygen $V_{p d}$, the oxygen-oxygen $V_{p p}$, etc.)

In this lecture we will consider the extension of this model which includes the long range Coulomb forces. This extension is necessary in order to examine the capability of strongly correlated electrons to participate in the screening and to determine the effects that strong local forces have on electronic plasmon. The second reason for the inclusion of the long range Coulomb forces is to stabilise the system against the phase separation, the instability that occurs in the part of the parameter space of the original $p$ - $d$ model. 
The introduction of long range Coulomb forces into the $p$ - $d$ model is relatively straightforward. However, a few points should be emphasized. First, as soon as long range Coulomb forces are considered, it is natural to extend the model from one plane to the three dimensional collection of parallel planes. The electron-electron interaction is described by

$$
H_{\text {coul }}=\frac{1}{2} \sum_{\vec{R}, \vec{R}^{\prime}, \alpha, \alpha^{\prime}} n_{\alpha}(\vec{R}) V_{\alpha \alpha \prime}\left(\vec{R}+\vec{a}_{\alpha}-\vec{R}^{\prime}-\vec{a}_{\alpha^{\prime}}\right) n_{\beta}\left(\vec{R}^{\prime}\right)
$$

where $\vec{R}=\left(R_{\|}, \vec{R}_{\perp}\right)$ represents the cell index and $\alpha$ denotes the orbital of the atom positioned at $\vec{a}_{\alpha}$ with respect to the origin of the unit cell. The potential $V_{\alpha \beta}\left(\vec{R}+\vec{a}_{\alpha}-\vec{a}_{\beta}\right)$, describes the interaction of electrons on different atoms in the unit cells separated by $\vec{R}$. It is proportional to $1 /\left|\vec{R}+\vec{a}_{\alpha}-\vec{a}_{\beta}\right|$ at long distances and reflects the relative position and the charge distribution in the orbitals at short distances. The Fourier transform, $V_{\alpha, \beta}(\vec{k})=\sum_{\vec{R}} V_{\alpha \beta}\left(\vec{R}+\vec{a}_{\alpha}-\vec{a}_{\beta}\right) \exp (i \vec{k} \vec{R}) \equiv$ $\tilde{V}_{\alpha \beta}(\vec{k}) \exp \left(-i \vec{k} \vec{a}_{\beta}-i \vec{k} \vec{a}_{\alpha}\right)$ behaves like $1 / \vec{k}^{2}$ at long wavelengths, while the short wave dependence reflects the structure and strength of the local forces. This may be emphasized by writing $\tilde{V}_{\alpha \beta}(\vec{k})$ in the form

$$
\tilde{V}_{\alpha, \beta}(\vec{k})=\frac{4 \pi e^{2}}{\left(a^{2} d_{\perp} \varepsilon_{\infty}\right) \vec{k}^{2}}+C_{\alpha \beta}(\vec{k})
$$

where $a^{2} d_{\perp}$ is the volume of the unit cell ( $a$ is the lattice constant of the $\mathrm{CuO}_{2}$ plane and $d_{\perp}$ is the distance between neighboring planes). The high frequency atomic screening is included through $\varepsilon_{\infty}$. The terms $C_{\alpha \beta}(\vec{k})$ have finite values at $\vec{k}=0$.

As usual, the $\vec{k}=0$ term of the $1 / \vec{k}^{2}$ part of the Hamiltonian cancels out because of the neutrality of the crystal. Further on, the 'passive' orbitals on the ions with fixed valence may be excluded from the electronic Hamiltonian, leaving only the active orbitals in the copper oxide planes in the model.f.

The Coulomb part of the $p$ - $d$ model, with the long range forces included, finally reads as

$$
H_{\text {coul }}=\sum_{\vec{k} \in B . Z ., \alpha, \beta} \frac{1}{2}\left[\left(1-\delta_{\vec{k}, 0}\right) \frac{4 \pi w t_{0}}{a^{3} \vec{k}^{2}}+C_{\alpha \beta}(\vec{k})\right] \tilde{n}_{\alpha}(\vec{k}) \tilde{n}_{\beta}(-\vec{k})
$$

\footnotetext{
${ }^{1}$ As in the original $p$ - $d$ model, we neglect the Fock terms here. Physically, this is correct for the long range, but not necessary correct for the short range Coulomb forces.

${ }^{2}$ A somewhat more complicated, but less suggestive form should be preferable for large $\vec{k}$, close to the zone boundary. For example, an additional $\exp \left(-\vec{k}^{2} a^{2}\right)$ factor may be introduced in order to make the cutoff at the zone boundary soft. Also, it is possible to replace $\vec{k}^{2}$ by $\sum_{l=x, y, z}\left[2-\cos \left(k_{l} d_{l}\right)\right] / d_{l}^{2}$ in order to have zero derivatives at the zone boundaries.

${ }^{3} \mathrm{It}$ is an instructive exercise to reformulate the calculation of the Madelung energy of some pure ionic crystal after the outlined procedure. The contribution of the $1 / \vec{k}^{2}$ term then explicitly cancels out due to the neutrality of the crystal, $\sum_{\alpha} n_{\alpha}=0$, and the Madelung energy per unit cell acquires the form $E_{M} / N=(1 / 2) \sum_{\alpha, \beta} C_{\alpha \beta}(0) n_{\alpha} n_{\beta}$.

${ }^{4}$ The terms neglected should be kept in mind as the source of a shift, presumably small, of the energy levels of active orbitals with doping. More important, these terms reappear as the electron-phonon coupling terms when lattice movement is considered.
} 
where $\tilde{n}_{\alpha}(\vec{k}) \propto \sum_{\vec{R}} n_{\alpha}(\vec{R}) \exp \left(-i \vec{k} \vec{R}-i \vec{k} \vec{a}_{\alpha}\right)$ and the dimensionless parameter $w=$ $e^{2} / d_{\perp} t_{0} \varepsilon_{\infty}$, measuring the strength of the long range Coulomb interaction is introduced. The approximate value for $w$ expected for cuprate superconductors may be estimated by taking $d_{\perp} \sim 12 \AA, \varepsilon_{\infty} \sim 3, t_{0} \approx 1.3 \mathrm{eV}$. This gives $w \sim 0.25$.

In the text that follows we will consider the situations with and without the $1 / \vec{k}^{2}$ term in the Hamiltonian. In the latter case, $C_{\alpha \beta}(\vec{k})$ corresponds to $U_{d}, V_{p d}$, $V_{p p}$ usually used in the literature. Physically, they represent the effective, screened electron-electron interaction. In the case where $1 / \vec{k}^{2}$ is explicitly included we continue to use the notation $U_{d}, V_{p d}, V_{p p}$, etc., although the physical meaning of $C_{\alpha \beta}$ 's in that case is somewhat different. They represent the local field corrections to the $1 / \vec{k}^{2}$ interaction, presumably large for short distances.

\section{Qualitative features of the electronic spectrum of doped charge transfer insulator}

Various estimates of the local Coulomb terms in the $p$ - $d$ model emphasize the large repulsion on the copper site, $U_{d} \sim 10 \mathrm{eV}$. The limit $U_{d} \rightarrow \infty$ is frequently considered in the literature, and we will work in that limit as well. The $n_{d} \leq 1$ constraint forced by infinite $U_{d}$ is often dealt with by introducing the auxiliary (slave) boson field $b$ and the auxiliary field fermion $f$ in terms of which the real electron operator on the copper site becomes the composite object, $d_{\vec{R}}=b_{\vec{R}}^{\dagger} f_{\vec{R}}$. The next step is usually to consider the mean field approximation for the boson field (the saddle point approximation in the partition function/path integral language). Usually, the corrections beyond the mean field (the Gaussian fluctuations around the saddle point) are also considered. While rigorous justification for this procedure is usually sought in the $1 / N$ expansion of the generalised model (i.e. $\mathrm{N}$ spin components instead of two), the physical appeal comes from the fact that it grasps some major physical features. In particular, this applies for related models 泊, that may be more accurately investigated by other means (for instance, the appearance of the Abrikosov-Suhl resonance in the Anderson model). For the $p$ - $d$ model this procedure qualitatively reproduces some basic experimental facts in cuprates as are the appearance of the in-gap resonance [6] and the large Fermi surface [7] on doping. A pedagogical survey of the method and some of its results may be found in ref. [8].

What basically happens at the mean field level [9] for the slave boson in the $p$ - $d$ model with large $U_{d}$ is: a) the boson field $b$ acquires a static component $B_{0}$ (with $\overline{n_{d}}=1-\left|B_{0}\right|^{2} \leq 1$ ) and an oscillatory component at the frequency $\lambda$; b) $\lambda$ also represents the shift of the copper orbital energy on changing from original electron to auxiliary fermion fields, $\varepsilon_{f}=\varepsilon_{d}+\lambda$; the copper-oxygen hybridization in the auxiliary fermion Hamiltonian is reduced with respect to the original hybridization, $t=t_{0} B_{0}$.

The band that occurs at $\varepsilon \approx \varepsilon_{f}$ ( $f$-band) in the auxiliary fermion density of states becomes the in-gap resonance when the real electron spectrum is considered. Quite simply, the majority of the spectral weight for the copper site that lies in the auxiliary fermion $f$-band is transfered back 10, 4 to original energy $\varepsilon_{d}=\varepsilon_{f}-\lambda$ on calculating the auxiliary fermion - auxiliary boson convolution in the real electron Green function $(-i)\left\langle T\left(b^{\dagger} f_{\sigma}\right)(t)\left(f^{\dagger} b\right)(0)\right\rangle$. The spectra are illustrated in Fig. 1 .

The Fermi energy stays in the resonance. The Fermi surface states are predom- 

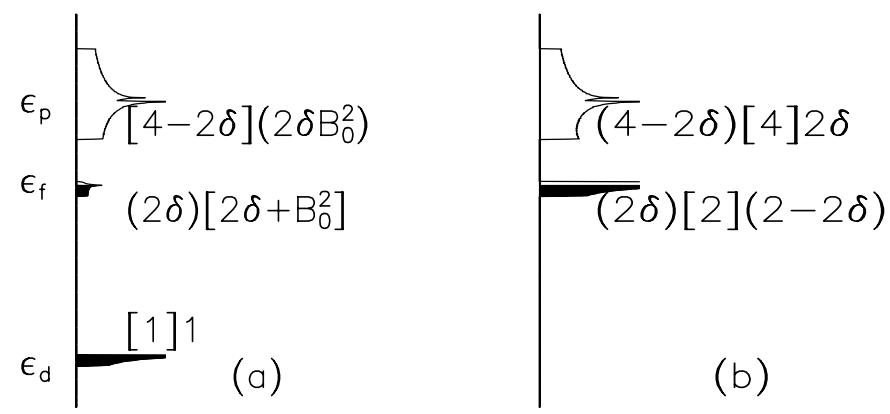

Figure 1: a) The density of states in doped $p$ - $d$ system, as obtained at the mean field level. b) The density of states for the auxiliary fermions. The labels $(p)[t](d)$ indicate the total weight of the particular part of the spectrum $[t]$ and the shares $(p)$ and $(d)$ of the oxygen and the copper orbitals, respectively. The resonance at $\varepsilon \approx \varepsilon_{f}$ in (a) and the corresponding band in (b) are approximately half filed for small doping level $\delta$. of the oxygen $(p)$ and the copper $(d)$.

inantly made of oxygen orbitals, especially in the doped charge transfer insulator $(\mathrm{CTI})$ regime $\left(\Delta_{p d} \gg t_{0}\right)$. In that regime the mean field value $B_{0}$ vanishes when the doping $\delta$ (measuring the hole concentration with respect to the level of one hole per cell) goes to zero, $B_{0}^{2} \propto \delta$. The renormalized auxiliary fermion bandwidth $\tilde{W} \propto B_{0}^{2}$, being also the effective width of the resonance, and the number of states in the resonance are also proportional to doping level $\delta$.

It will be useful to keep this qualitative picturef in mind when the results for the charge susceptibilities are discussed.

\section{Charge transfer susceptibilities and Coulomb screening}

The results for the charge susceptibilities that we present are calculated on taking into account the first fluctuation correction beyond the mean field approximation. While for large $U_{d}$ the slave boson approach is used, for other, smaller Coulomb terms we use the Hartree approximation [8, 12] as the mean field approximation. The fluctuation correction for the long range Coulomb interaction corresponds to the usual random phase approximation (RPA). Fig. 2 reflects the simple structure of our calculations. The charge transfers that we will consider may be expressed in terms of the copper and the oxygen site charges $n_{d}, n_{x}, n_{y}$. The combinations reflecting different charge transfer symmetries and different physics are $n_{p}=n_{x}+n_{y}$ (the charge on oxygen sites), $n_{p p}=n_{x}-n_{y}$ (the charge transfer between inequivalent oxygen sites), $n_{p d}=n_{p}-n_{d}$ (the copper-oxygen charge transfer) and $n_{n}=n_{p}+n_{d}$ (the total charge in the cell). The susceptibilities $\chi_{\alpha}=\left\langle\left\langle\tilde{n}_{\alpha} \tilde{n}_{\alpha}\right\rangle\right\rangle, \alpha=d, p, p p, p d, n$

\footnotetext{
${ }^{5}$ This picture may be refined by calculating the auxiliary field propagators beyond the saddle point level [4, 8, 11] and by calculating the electronic spectrum beyond the decoupling approximation, leading to simple convolution.

${ }^{6}$ The Hartree approximation and the RPA fluctuation correction does not seem to provide an adequate treatment for the short range Coulomb interaction terms as is the $V_{p d}$ term. However, we will really consider only some qualitative features that these interaction bring in. These features are expected to remain present even if some more adequate treatment like the full Hartree-Fock approximation is used.
} 


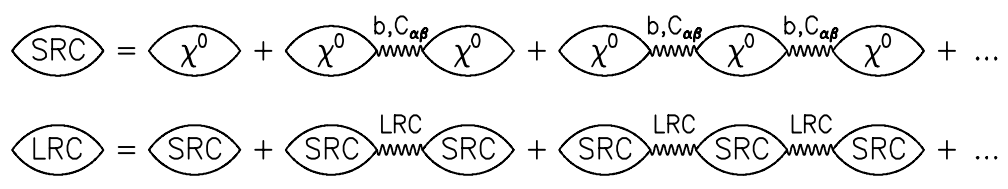

Figure 2: The corrections beyond the saddle point and Hartree approximations.

will be considered. We will distinguish the susceptibilities $\chi^{0}$ of the tight binding fermions with the mean field renormalized band parameters; the susceptibilities $\chi^{S}$ calculated for the model with short range forces; the susceptibilities $\chi$ calculated for the model in which the long range Coulomb forces are included as well.

In Figs. 3 various static charge transfer susceptibilities are shown as a function of the wave vector $\vec{k}=(k, 0,0)$ (hereafter we will use the bare copper-oxygen hybridization energy $t_{0}$ as the energy unit and the $\mathrm{CuO}_{2}$ plane lattice constant $a$ as the unit distance in order to simplify the notation). From these figures the following
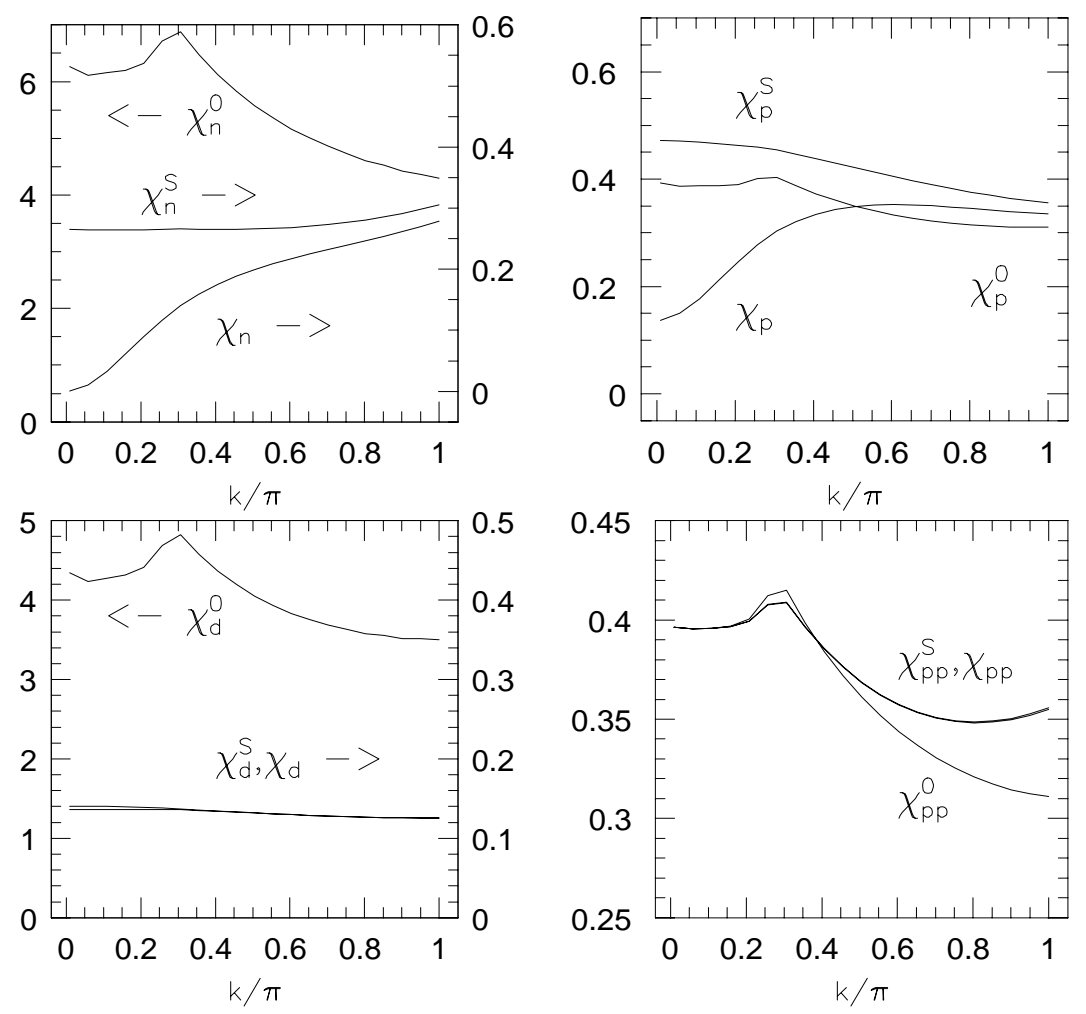

Figure 3: Static charge susceptibilities for $\Delta_{p d}=4, \delta=0.1$ as functions of the wavevector, $\vec{k}=(k, 0,0)$ : Shown susceptibilities reflect the transfers of a) the total cell charge b) total oxygen charge in the cell c) the copper charge d) the intracell oxygen-oxygen charge transfer.

may be noted:

a) The inclusion of the fluctuation of the slave boson field, corresponding to $U_{d}=$ $\infty$, suppresses the charge fluctuation on the copper sites for all wavevectors: $\chi_{d}^{0} \gg \chi_{d}^{S}$. The copper-oxygen charge transfer susceptibility is also diminished. It can be also seen that the Fermi surface enhancement] seen in $\chi_{d}^{0}$ at $k_{x} \approx 0.1 \pi$

\footnotetext{
${ }^{7}$ Several types of the Fermi surface enhancements of the static susceptibilities in the two dimen-
} 
is lost in $\chi_{d}^{S}$.

b) The fluctuation of the oxygen charge $n_{p}$ is not so much affected by the auxiliary boson fluctuations. However, the Fermi surface effect disappears in $\chi_{p}^{S}$ as well.

c) The inclusion of the long range Coulomb forces further suppresses the total charge fluctuations at small wavevector, $\chi_{n} \ll \chi_{n}^{S}$. While this is expected to happen, it is important to realize that the charge fluctuation on the oxygen sites are mostly affected. This implies that the holes in the oxygen orbitals dominantly participate in the Coulomb screening and that their dynamics determines the dielectric properties of the system. This issue will become more clear when we consider the charge fluctuation spectra.

d) The $p p$ susceptibility related to the charge transfer of the between inequivalent oxygen atoms ( $x$ and $y$ ) in the unit cell is unaffected both by the long range Coulomb interaction and by the slave boson fluctuations. The reason lies in the particular symmetry of the $p p$ charge transfer. In particular, the Fermi surface effects are pronounced in $\chi_{p p}^{S}$ and $\chi_{p p}$, in contrast to the their absence in the susceptibilities $\chi_{p}^{S}$ and $\chi_{p}$ related to the total oxygen charge. In fact, all van Hove and nesting enhancements present in the tight binding's $\chi^{0}$ remain in $\chi_{p p}$ whereas they are suppressed in all other channels by the large $U_{d}$. This, in particular, means that the $\vec{k}=0$ charge transfer transitions [3, 13, 14] and $\vec{k}=(\pi, \pi)$ charge density wave should be searched for mainly in the $p p$ channel. The doping level particularly favorable is the one for which the Fermi surface touches the zone boundary.

The dependence of the static dielectric function on the wavevector is shown in Fig. 4 . The behaviour $1 / \varepsilon(k) \propto \vec{k}^{2} \rightarrow 0$ as $\vec{k} \rightarrow 0$ corresponds to the full metallic screening. More important, it should be noted that the screening distance, expressed as $1 / k_{s}$ in Thomas-Fermi language, where $1 / \varepsilon(k)=\vec{k}^{2} /\left(\vec{k}^{2}+k_{s}^{2}\right)$, is of the order of the lattice spacing, in spite of the fact that the concentration of doped holes is small (average distance between them is approximately three lattice constants for $\delta=0.1$ ). The doping dependence of the screening length shows no drastic changes with doping. This reflects the fact that density of states of the in-gap resonance (i.e., (number-of-states)/(resonance-width)) does not change very much with doping, even in the doped CTI regime.

\section{Dynamic correlation functions and collective modes}

More detailed information on the charge dynamics are obtained on considering the dynamic charge correlation functions. They further clarify which orbitals participate in various intra and inter-cell charge transfers and how they are affected by short and long range Coulomb forces. Some aspects of the the spectrum of charge fluctuations for the system with large $U_{d}$ was considered by Kotliar, Castellani and coworkers [8, 15]. They discussed the appearance of the high frequency exciton collective mode $\left(\omega=\omega_{p d}\right)$ as well as the zero sound mode in the large $U_{d} p$ - $d$ model. The spectrum

sional tight binding model occur at $\vec{k} \approx 0$ and $\vec{k} \approx( \pm \pi, \pm \pi)$ : van Hove effects, as well as the nesting or the sliding Fermi surface effects, if some flat parts of the Fermi surface exist. The degeneracy of these effects for the square Fermi surface is lifted for some more complicated Fermi surface shapes. 

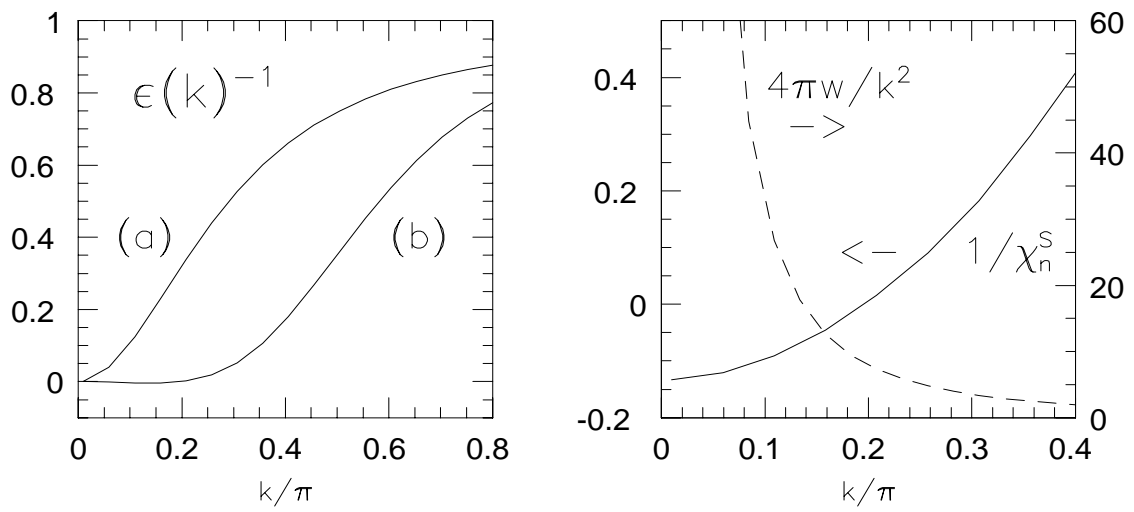

Figure 4: The figure on the left shows the dielectric function for (a) $V_{p d}=0$ and (b) $V_{p d}=1.25$. The second case (as discussed in section 6.) is rather close to the border of the 'normal' $\left(V_{p d}<1.15\right)$ and FEPSI $\left(V_{p d}>1.15\right)$ situation. The figure on the right shows the total charge susceptibility in the unstable $V_{p d}=1.25$ system without the long range forces, $\chi_{n}^{S}(0)<0$. Other parameters are: $\Delta_{p d}=4, \delta=0.1, V_{p p}=0$.

of the oxygen charge fluctuations shown in Fig. 5 the appearance of these modes in the spectrum together with the intraband and interband continua. Fig. 51)
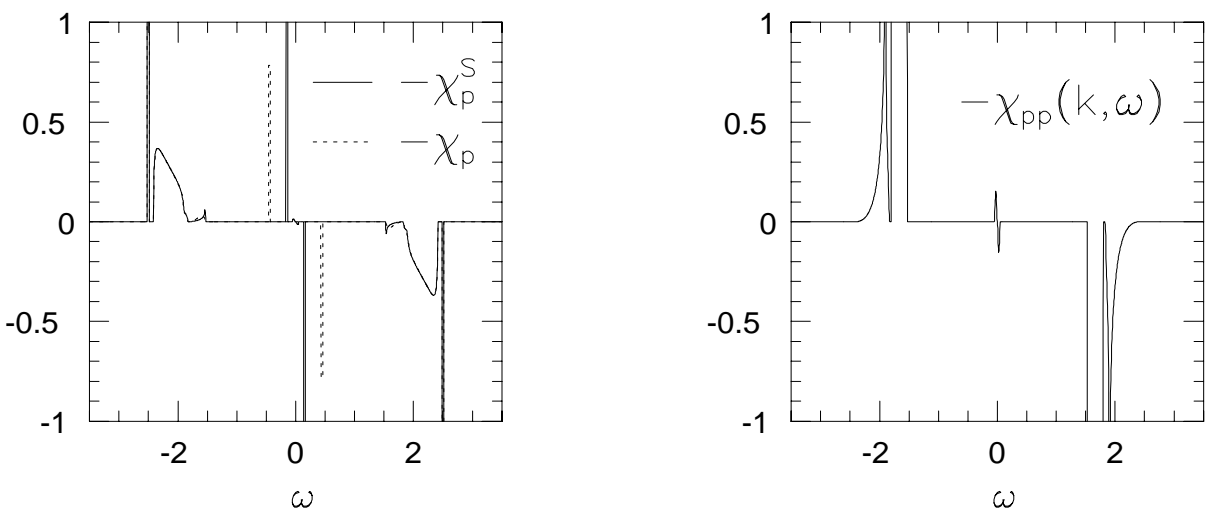

Figure 5: Left: The spectrum of the fluctuation of the charge in the oxygen orbitals, $n_{p}=n_{x}+n_{y}$. The full line correspond to the case without long range interaction. The intercell and the intra-cell $(\mathrm{Cu}-\mathrm{O})$ charge fluctuations, corresponding to the zero sound mode and the $\mathrm{Cu}-\mathrm{O}$ exciton mode, and may be distinguished. The long range Coulomb interaction changes the zero sound to the intraband plasmon mode, pushing its frequency to finite frequency at low wavelengths. Right: In the intra-cell oxygen-oxygen charge fluctuation spectrum, $n_{p p}=n_{x}-n_{y}$, there are no collective modes present. The strength of the interband contribution in $\chi_{p p}$ is big relative to those in other spectra. Parameters as before. $V_{p d}=0, \vec{k} / \pi=(0.11,0,0)$.

also shows the effect of the long range Coulomb interaction on the spectra. It is interesting to note that the frequency of the exciton mode is not at all affected at long wavelengths. This shows the quadrupolar character of the corresponding charge excitations. On the other hand, zero sound mode changes to the intraband plasmon mode with the dispersion $\omega_{1}^{2}(\vec{k})=\omega_{1 p}^{2} \vec{k}_{\|}^{2} /\left(\vec{k}_{\|}^{2}+k_{\perp}^{2}\right)$ usual for quasi-2d systems with negligible inter-plane hopping. The frequency (as well as the strength of the corresponding pole in the density-density correlation function) vanishes for 

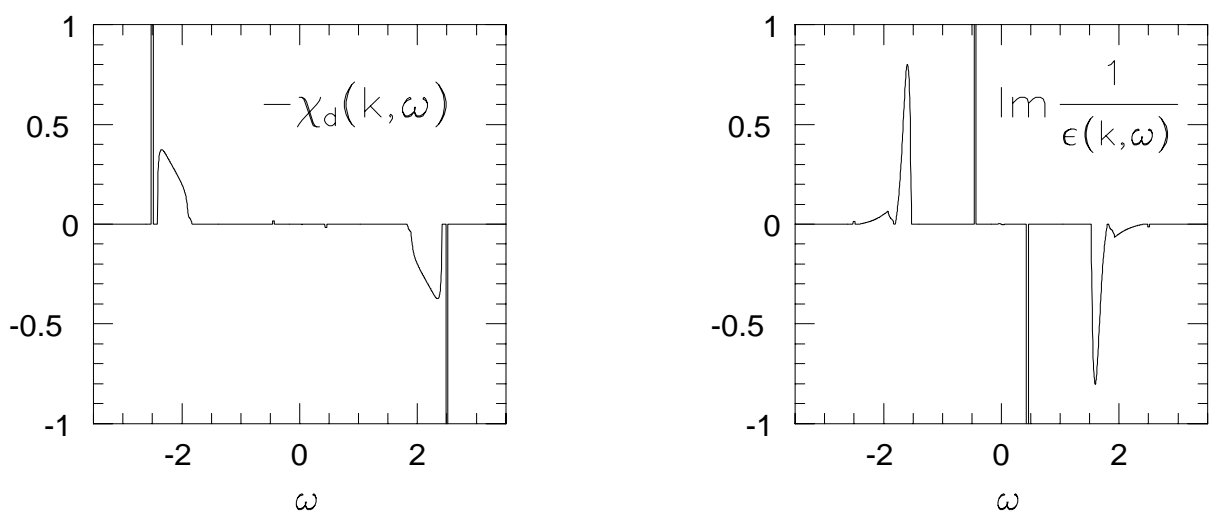

Figure 6: Left: The spectrum of charge fluctuations on the copper site. The Cu-O exciton mode and the interband excitations are pronounced. Right: The total charge fluctuation spectrum as seen through $1 / \varepsilon(\vec{k}, \omega)$. The intraband plasmon and the intra-cell dipolar excitation, which lies within the interband continuum, dominate the spectrum.

the wave vector directed perpendicular to the planes. The frequency $\omega_{1 p}$ of the long wavelength in-plane oscillations increases on increasing the strength $w$ of the long range Coulomb interaction, but always stays in the gap. The dependence on $w$ may be understood through a simple equation for the Coulomb collective modes in the two (infinitely narrow) band systems,

$$
1-\frac{\Omega_{1}^{2}}{\omega^{2}}-\frac{\Omega_{2}^{2}}{\omega^{2}-\Delta_{p f}^{2}}=0 .
$$

Here $\Delta_{p f}=\varepsilon_{f}-\varepsilon_{p}$ measures the renormalised gap while $\Omega_{1}^{2}$ and $\Omega_{2}^{2}$ are products of the Coulomb factor $w \propto e^{2}$ and the intraband and the interband oscillator strengths, respectively. $\Omega_{1}$, reflecting the intraband charge transfer oscillator strength, assumes particularly simple doping dependence $\Omega_{1}^{2} \propto \delta$ in the doped CTI regime. This is indeed expected from the picture that we have developed until now.

The second solution of the equation (corresponding to the third collective mode in the calculation) represents the intracell dipolar mode. It starts from finite frequency $\omega \approx \Delta_{p f}$ for small $w$ and becomes the 'big plasmon' as $w$ is further increased. However, in our calculations the substantial $w>1$ is required to push the frequency of this mode above the interband excitation continuum.

In more detailed inspection of the spectra $\chi_{\alpha}^{\prime \prime}(\vec{k}, \omega) \equiv \operatorname{Im} \chi_{\alpha}(\vec{k}, \omega)$ of various charge transfer modes, we find that the intraband part of the spectrum is suppressed in all channels, except from the $p p$ mode. The zero sound/plasmon mode dominates in the low frequency part of spectra of the total charge fluctuation $\chi_{n}^{\prime \prime}(\vec{k}, \omega)$ and the oxygen charge fluctuation $\chi_{p}^{\prime \prime}(\vec{k}, \omega)$. The strength of this mode in the channels related to long wavelength charge fluctuations on the copper site is rather small. These spectra are dominated by the interband excitations and the quadrupolar exciton mode. The later is absent in the total charge fluctuations at long wavelengths. The imaginary part of the inverse dielectric function. 9 is shown in Fig. 6.

\footnotetext{
${ }^{8}$ None of the collective modes appears in $\chi_{p p}^{\prime \prime}(\vec{k}, \omega)$ for $\vec{k}=0$. For finite $\vec{k}$ their strength is negligible.

${ }^{9}$ We extract the 'macroscopic' dielectric function from the matrix of charge susceptibilities by considering the coupling of the system to the external potential.
} 


\section{$6 \quad$ Frustrated electronic phase separation instability (FEPSI)}

The dynamics and the dielectric properties of the system with frustrated electronic phase separation instability is even more interesting to consider. Experimentally, the proximity of the phase separation instability (PSI) and superconductivity in cuprates and related materials is rather well documented [16]. The possibility for PSI in the model with strong electron-electron interaction was addressed by several authors. In particular, V.J. Emery and S.A. Kivelson considered the case of low doped antiferromagnet and discussed the possible consequences of FEPSI for the normal state properties and superconductivity in cuprates [17.

The phase separation instability within the $p$ - $d$ model with short range forces occurs as the copper-oxygen Coulomb term $V_{p d}$ is increased in the hole doped system, as pointed out in ref. [12]. However, the enhancement of the copper-oxygen charge transfer by $V_{p d}$, often emphasized by some authors [1, 2], is not an important issue here.ण丁口

The main reason for a system with strong correlations to choose the phase separated phase is to diminish its kinetic energy. The kinetic energy diminishes on doping since the frustration for the electron hopping caused by strong coulomb interaction becomes less effective ${ }^{\Gamma}$. On approaching the point of thermodynamic instability the derivative $(\partial \mu / \partial n)=(\partial \mu / \partial \delta)$ (the rate of change of the chemical potential with doping) approaches zero and becomes negative in the unstable phase. Also, the susceptibility $\chi_{n}^{S}\left(\vec{k}=0, \omega=0\right.$ ) (as well as all other $\chi_{\alpha}^{S}$ 's) diverges at this point, $1 / \chi_{n}^{S}(\vec{k}=0, \omega=0)=(\partial \mu / \partial n)$. An example of $1 / \chi_{n}^{S}(k)$ in the unstable system is shown in Fig. 4 .

Of course, once the long range Coulomb interaction is introduced into the model, the true phase separated state is not likely to appear because of the huge costs in the Coulomb energy. Speculating on this issue, various authors stop at this point and suggest that the charge density wave (CDW) state will replace the phase separated state (i.e. that the instability will show as the divergence of $\chi_{n}$ at some finite wavevector instead of at $\vec{k}=0$ ). However, the pure electronic CDW (assuming that the lattice is too rigid to participate in the formation of the CDW) corresponds to unrealistically weak Coulomb forces for the cuprate superconductors, $w \ll 1$. We find that the stable FEPSI state with $1 / \chi_{\alpha}(\vec{k})>0$ and homogeneous electronic density is more probable. However, the static dielectric function,

$$
\frac{1}{\varepsilon(\vec{k})}=\frac{1}{1+\left(4 \pi w / \vec{k}^{2}\right) \chi_{n}^{S}\left(\vec{k}_{\|}\right)}
$$

is negative in FEPSI systems at long wavelengths since $\chi_{n}^{S}(\vec{k})$, which accounts for the local forces, turns negative, $1 / \chi_{n}^{S}(0) \propto(\partial \mu / \partial n)<0$, as shown in Fig. 1 .

\footnotetext{
${ }^{10}$ The value of $V_{p d}$ for which the enhancement of the copper-oxygen charge fluctuation takes place (possibly related to the softening of the corresponding exciton mode) is much bigger than the value required for PSI. For example, in our calculations for $\Delta_{p d}=4 t_{0}$ PSI occurs already at $V_{p d}=1.15 t_{0}$, while a value approximately two times bigger is required for the cooper-oxygen charge transfer instability to happen.

${ }^{11}$ The $p$ - $d$ model in the parameter range $\left(U_{d}-\Delta_{p d}\right) \ll \Delta_{p d} \ll U_{d}$ exhibits the phase separation instability 10 even for $V_{p d}=0$.

${ }^{12}$ V.J. Emery originally discussed and repeatedly emphasized this issues in the framework low doped $t$ - $J$ model.
} 
The figure suggests the following formula to describe the dependence of the dielectric function on the wavevector,

$$
\frac{1}{\varepsilon(k)} \approx \frac{1}{1-b}\left(\frac{k^{2}}{k^{2}+k_{s 1}^{2}}-\frac{k^{2} b}{k^{2}+k_{s 2}^{2}}\right),
$$

with $1 / k_{s 2} \ll k_{s 2}\left(1 / k_{s 2}\right.$ is at least of the order of few lattice constants in the $\mathrm{CuO}_{2}$ plane) and $b<1$. The formula resembles the Thomas-Fermi formula, but has two characteristic wavevectors instead of one. In order to get a qualitative feeling for the system, one may try to play with a three dimensional toy model with the dielectric function of the form (77). The feature that readily emerges is the overscreening of the test charge at distances $1 / k_{s 1}$ followed by the complete screening at distances beyond $1 / k_{s 2}$. This implies the attraction of two equal test charges down to distances $1 / k_{s 2}$. Also, the limit $1 / \varepsilon \rightarrow 0$ as $k \rightarrow 0$ accounts for the total metallic screening. It is easy to see that a piece of the material exposed to the static electric field behaves just like an ordinary metal, developing a finite surface charge in order to ensure $\vec{E}=0$ in the bulk.

Returning to our quasi-two dimensional FEPSI metal we examine its static charge susceptibility. It is given in a form that may be easily analysed (see again Fig. (1),

$$
\frac{1}{\chi_{n}(\vec{k})}=\frac{4 \pi w}{k_{\perp}^{2}+\vec{k}_{\|}^{2}}+\frac{1}{\chi_{n}^{S}\left(\vec{k}_{\|}\right)}
$$

The most pronounced features (see Fig. 7) are the maximum at finite $k_{\|}=k_{c}$ (not related to some particular Fermi surface wave vector $-k_{c}$ decreases with decreasing $w)$ for $k_{\perp}=0$, and the increase of $\chi_{n}$ towards the $k_{\perp}=\pi / d_{\perp}$ zone boundary. It may be noted here that the divergence of $\chi_{n}\left(k_{\|}=k_{c}\right)$ for sufficiently small $w$ is the usually mentioned CDW instability which comes as the alternative to the FEPSI homogeneous in the $\mathrm{CuO}_{2}$ plane. This instability corresponds to the softening of the intraband plasmon branch at $k_{\|}=k_{c}$. However, the $\mathrm{Cu}-\mathrm{O}$ exciton mode is not affected at this point.
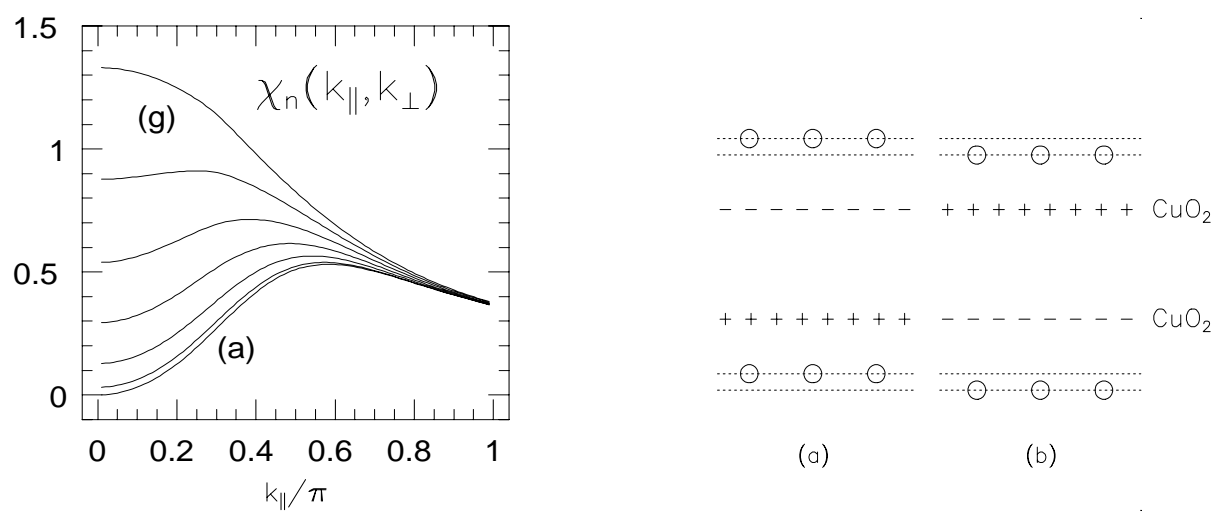

Figure 7: Left: The dependence of the total charge susceptibility on $k_{\perp}, k_{\perp} / \pi=$ $0(a), 0.1, . ., 0.6(g)$. Right: The possible effect of large inter-plane charge transfer susceptibility on apex oxygen atoms in the systems with $\mathrm{CuO}_{2}$ bilayers, like $\mathrm{YBa}_{2} \mathrm{Cu}_{3} \mathrm{O}_{7-x}$. 


\section{$7 \quad$ Lattice properties and the stability of FEPSI systems}

Finally, we may turn to the question of the stability of the FEPSI system, considering in particular the influence of the electronic gas on the ionic lattice (which was considered infinitely rigid up to now). First, the question of total compressibility of the FEPSI system may be addressed. It may be shown in a number of ways that the short range forces between ions provide the stability of the system. One way is to consider the longitudinal sound velocity and the corresponding frequency $\omega^{2}(\vec{k})=c^{2} \vec{k}^{2}=c_{O}^{2} \vec{k}^{2}+\Omega_{p, i o n}^{2} / \varepsilon_{e l}(k)$ consisting of the contribution of the short range forces between ions and the long range forces screened by electrons. For FEPSI systems the second term is negative. In terms of the total bulk modulus $B$ which determines the sound velocity $c^{2}=B / \rho$, the contribution of the second term equals $n^{2}(\partial \mu / \partial n)<0$. This, however, does not jeopardize the stability of the system, since size of the the electronic contribution is two orders of magnitude smaller than the total bulk modulus measured for cuprates, [3] $B \sim 10^{-12} \mathrm{dyn} / \mathrm{cm}^{2}$. The reason for small absolute value of the electronic contribution may be ultimately traced back to the small density of the electronic gas ${ }^{\text {T4 }}$

More interesting than the compression of the whole system are the phonon modes that couple directly to the charge fluctuations that our model calculations distinguishes as relatively big. In summary ${ }^{\boxplus}$, these are: a) the in-cell oxygen-oxygen charge fluctuations b) the $k_{\perp}=\pi$ inter-plane charge transfer (this becomes $k_{\perp}=0$ when systems with $\mathrm{CuO}_{2}$ bilayers are considered) c) the incommensurate charge density fluctuations inside the $\mathrm{CuO}_{2}$ planes. These modes may be expected to become soft $\tilde{\omega}_{p h}^{2}=\omega_{p h, 0}^{2}-g_{e p}^{2} \chi_{e l}$ when the electron-phonon coupling $g_{e p}$ is substantial or lattice instabilities and/or anharmonicities may result.

In that respect it is interesting to note that, indeed, in several copper oxide superconductors anomalies in the lattice modes coupled to the intracell oxygenoxygen charge transfer are observed. For example, in $\mathrm{Tl}_{2} \mathrm{Ba}_{2} \mathrm{CaCu}_{2} \mathrm{O}_{8}$ in the normal state the dominant mode for the in-plane oxygen motion is one that couples to $n_{p p}$. This kind of oscillation becomes suppressed [19] in the superconducting phase. Similar competition of the superconductivity and lattice deformation that couples to $n_{p p}$ is found 20, 3] in the LTT phase of $\mathrm{La}_{2-x} \mathrm{Ba}_{x} \mathrm{CuO}_{4}$.

The discussion on the experimental evidence for the in-plane incommensurate CDW in related, but non-superconducting compounds, may be found in ref. [17.

Here we would like to point out one possible sign of the FEPSI situation in

\footnotetext{
${ }^{13}$ The correct order of magnitude for the crystal compressibility may by be obtained from simple Madelung calculations.

${ }^{14}$ In the jellium language, $r_{s} \sim 8$, if we count one hole per unit cell; counting only doped holes we get $r_{s} \sim 16$ for $\delta=0.1$. At this point, it seems interesting to note that the theoretical search for the high temperature superconductivity before the discovery of cuprate superconductors pointed, indeed, towards the low density metals 18$]$. One of the basic properties of these systems is the appearance of the negative static dielectric function. That negative static dielectric function is the feature desirable for the electronic mechanism of superconductivity was rather pedagogically explained by Littlewood in ref. [2]. However, as emphasized by Kirzhnits at al. [18], this is not the sufficient condition for the superconductivity to occur - the interaction between test charges is not the one that enters the gap equation - the local field corrections and correlation effects that themselves lead to negative static dielectric function should be taken care of, as well.

${ }^{15}$ The possibility of soft $\mathrm{Cu}-\mathrm{O}$ charge transfer exciton is out of focus of this lecture. However, the formalism set here is perfectly suitable for its consideration since, as already explained, one almost inevitably works 'deeply' inside the FEPSI phase.
} 
$\mathrm{YBa}_{2} \mathrm{Cu}_{3} \mathrm{O}_{7-x}$, the compound which contains two $\mathrm{CuO}_{2}$ layers per unit cell. From our discussion we expect to find anomalies for ion movements that couple to the charge transfer between these layers. The IR-active mode of the apex oxygen atoms is a natural candidate for such coupling. The coupling, being strong enough, results in the situation with two equivalent, minimum energy positions for the apex oxygen atoms (see Fig. 0 for illustration). Experimentally, this picture, supported later on by some other measurements, first emerged from the analysis of the EXAFS [21] in $\mathrm{YBa}_{2} \mathrm{Cu}_{3} \mathrm{O}_{7-x}$

\section{Conclusion}

The strong short range Coulomb forces substantially complicate the charge dynamics in doped copper-oxide planes. It seems difficult to envisage some effective band picture which would simultaneously account for the static $\left(k_{s}\right)$ and dynamic $\left(\omega_{p}\right)$ screening as well as some Fermi surface effects that we pointed out. Also, the strong Coulomb forces may tend to cause electronic phase separation, the vicinity of which was pointed out by several authors as the possible source of superconductive pairing and the anomalous normal state properties. At this point the introduction of the long range Coulomb forces into the model seems crucial, what we did for the $p$ - $d$ model. We showed that, while the long range Coulomb forces suppresses the instability, the negative static dielectric function and, more important, rather big total charge susceptibilities in some parts of the $k$-space remain as the characteristic signs of

FEPSI systems. Some of these signs were found in cuprate superconductors and related materials. While the lattice instabilities which may occur in order to exploit the large electronic susceptibilities probably do not contribute to the appearance of superconductivity, the effective electronic interaction which drives FEPSI may be favorable in that sense.

Acknowledgements. We gratefully acknowledge the discussions and remarks by I. Batistić and D. K. Sunko.

\section{References}

[1] C.M. Varma, S. Schmitt-Rink, E. Abrahams, Solid State Commun. 62 (1987) 681; C.M. Varma et al., Phys. Rev. Lett 63 (1989) 1996; C.M. Varma, Phys. Rev. Lett 75 (1995) 898;

[2] P.B. Littlewood in: V.J. Emery(ed.), Correlated electron system, World Scientific 1993., $p .1$

[3] S. Barišić in: B.K. Chakraverty (ed.), Critical Trends in High $T_{c}$ Superconductivity, World Scientific, 1991.

[4] P. Coleman, Phys. Rev. B 35 (1987) 5072

[5] V.J. Emery, Phys.Rev. Lett. 58 (1987) 2794

[6] N. Nucker et al., Phys.Rev. B 37 (1988) 5158; T. Takahashi et al., Nature 334 (1988) 691; T. Watanabe et al., Phys.Rev. B 44 (1991) 5316

[7] J.C. Compuzano et al., Phys.Rev.Lett. 64 (1990) 2308; D.S. Dessau et al., Phys. Rev. Lett. bf 71 (1993) 2781; Aebi P. et al., Phys. Rev. Lett. 72 (1994) 2757

[8] G. Kotliar in: V.J. Emery (ed.), Correlated electron system, World Scientific 1993., $p$. 118

[9] G. Kotliar, P.A. Lee, N. Read, Physica C 153-155 (1988) 538 
[10] E. Tutiš, Ph.D. thesis, University of Zagreb, 1994.

[11] H. Nikšić, E. Tutiš, S. Barišić, Physica C. 241 (1995) 247-256

[12] M. Grilli, R. Raimondi, C. Castellani, C. Di Castro, G. Kotliar, Phys.Rev.Lett. 67 (1991) 259

[13] S. Barišić, J. Zelenko, Solid State Commun. 74 (1990) 367

[14] S. Barišić, E. Tutiš, Solid State Commun. 87 (1993) 557

[15] C. Castellani, G. Kotliar, R. Riamondi, M. Grilli, Z.Wang, M. Rozenberg, Phys. Rev. Lett 69 (1992) 2009

[16] K.A. Müller and G. Bedenek (eds.), Phase Separation in Cuprate Superconductors, World Scientific, 1993

[17] V.J. Emery and S.A. Kivelson, Physica C 209 (1993) 567; C 235-240 (1994) 189

[18] O.V. Dolgov, D.A. Kirzhnits and E.G. Maksimov; V.L. Ginzburg and D.A. Kirzhnits in: V.L. Gizburg (ed.), Superconductivity, Superdiamagnetism, Superfluidity, Mir, Moscow 1987.

[19] B.H. Toby, T. Egami, J.D. Jorgensen, M.A. Subramanian, Phys.Rev.Lett. 64 (1990) 2414

[20] M. Sera, Y. Ando, K. Fukuda, M. Sato, I. Watanabe, S. Nakshima, K. Kumgai, Solid State Commun. 69 (1990) 851

[21] J. Mustre de Leon et al., Phys. Rev. Lett. 65, (1990) 1675; Phys. Rev. B 45, (1992) 2447 